\title{
Capillary Depressions for Nearly Planar Menisci
}

\author{
M. C. I. Siu \\ Institute for Basic Standards, National Bureau of Standards, Washington, D.C. 20234
}

(July 17, 1969)

\begin{abstract}
It is demonstrated analytically that the capillary depression $C$ of a nearly planar meniscus is linearly related to its meniscus height $h$ by the relationship $C=h /\left[1-I_{0}(r / a)\right]$. Here, $a^{2}=\gamma / \rho g, \rho$ and $\gamma$ are the density and surface tension of the liquid respectively, $g$, the acceleration due to gravity, $r$ is the radius of the capillary tube at the liquid-solid-gas interface, and $I_{0}(z)$ is the modified Bessel function of the first kind and zero order.
\end{abstract}

Key words: Bessel functions; calculus of variations; capillary depression; Laplace's equation; meniscus; meniscus height; nearly planar meniscus.

\section{Introduction}

Corrections due to capillarity must be known or eliminated in accurate manometric procedures. The problem of capillary depression (or rise) for arbitrarily shaped gas-liquid interfaces has been studied extensively, both theoretically and experimentally. Little attention, however, has been given specifically to nearly planar menisci occurring in large bore precision mercury manometers and which have been produced by Moser and Poltz [1] ${ }^{1}$ in the compression capillary of a McLeod gage to reduce errors due to capillarity.

It is well known $[2,3,4]$ that a finite pressure difference exists across a gas-liquid interface whenever it is not planar. Laplace [2] was the first to show theoretically that such local pressure differences were solely determined from the mean curvature of the surface and the surface tension of the liquid. This is a statement of Laplace's well-known nonlinear differential equation for liquid manometric work. Numerical solutions [3, 4, 5, 6] of Laplace's equation exist and tables showing the dependence of capillary depressions on meniscus heights and tube bores have been constructed. Values appearing in these tables are for arbitrary menisci, and interpolation is frequently required when using them.

In this work only nearly planar menisci are considered, and their associated capillary depressions are derived analytically.

\section{Theory}

The method of the calculus of variations [7] has been used by Goodrich [8] in his study of the static meniscus. This treatment of the static meniscus is similar to that

${ }^{1}$ Figures in brackets indicate the literature references at the end of this paper. of Goodrich in that the calculus of variations is applied.

Figures la and $\mathrm{lb}$ represent the cross section of portions of capillary systems which are capable of producing nearly planar menisci which are of capillary dimensions [1]. The coordinate systems and variables used in this discussion are shown in these figures. In these figures $\mathrm{LMN}$ and $\mathrm{L}^{\prime} \mathrm{M}^{\prime} \mathrm{N}^{\prime}$ represent portions of the vessel. $\mathrm{MO}^{\prime} \mathrm{M}^{\prime}$ represents the liquid-gas interface. $\mathrm{OO}^{\prime} \mathrm{O}^{\prime \prime}$ is taken to be the axis of symmetry of the meniscus. The $x y$-plane is perpendicular to $\mathrm{OO}^{\prime} \mathrm{O}^{\prime \prime}$ with

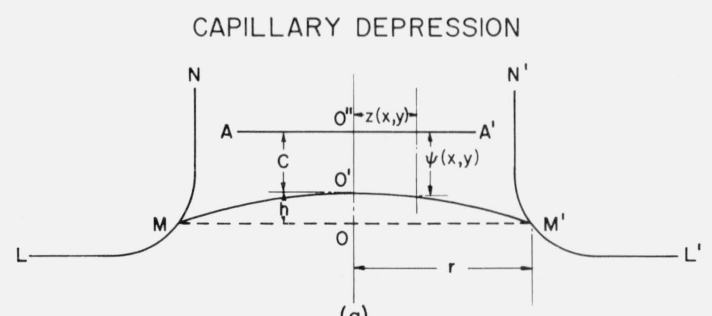

(a)

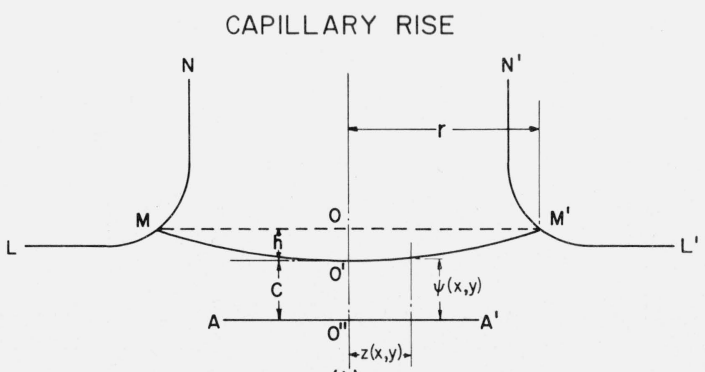

(b)

FIGURE 1. Coordinate system and displacements for a nearly planar meniscus. 
$z(x, y)=\sqrt{x^{2}+y^{2}}$ and $\theta=\tan ^{-1} y / x$ (not shown in these figures). This distance from $\mathrm{OO}^{\prime}$ to the gas-liquidsolid interface is $r$. $\mathrm{AA}^{\prime}$ represents the position the meniscus would take in the absence of capillarity. All displacements $\psi(x, y)$ are taken with respect to $\mathrm{AA}^{\prime}$.

Let $w_{g}$ and $w_{\gamma}$ be energies associated with the actions of gravity and surface tension, respectively. Consider the meniscus generated from the rotation of the segment $\mathrm{O}^{\prime} \mathrm{M}^{\prime}$ about $\mathrm{OO}^{\prime}$. For convenience of discussion, consider the situation shown in figure lb. The potential energy of a column of liquid of unit cross-sectional area and height $\psi(x, y)$ is given by

$$
w_{g}=\frac{1}{2} \rho g \psi^{2}(x, y),
$$

where $\rho$ is the density of the liquid, $g$ is the acceleration due to gravity. The surface element $z d \theta d \zeta$, where $d \zeta$ is an arc length measured along $\mathrm{O}^{\prime} \mathrm{M}^{\prime}$, possesses a surface free energy of amount $\gamma z d \theta d \zeta$, where $\gamma$ is the surface tension of the liquid. This exceeds that of a planar element $z d \theta d z$ by amount $w_{\gamma}=\gamma z d \theta(d \zeta-d z)$. Elementary geometrical considerations give

$$
d \zeta=d z \sqrt{1+|\nabla \psi|^{2}}
$$

where $\nabla \psi=(\partial \psi / \partial x, \partial \psi / \partial y)$. Thus the excess surface free energy per unit area, i.e., $z d \theta d z=1$ is given by

$$
w_{\gamma}=-\gamma\left[1-\sqrt{1+|\nabla \psi|^{2}}\right] .
$$

The total energy of the static meniscus is taken to be

$W[\psi(x, y)]=\iint_{R}\left(w_{g}+w_{\gamma}\right) d x d y+\int_{\Gamma} \phi(s) d s$.

The second term on the right is a line integral term and has been included to take into account any energy associated with the liquid-gas-solid interface along the curve $\Gamma: x^{2}+y^{2}=r^{2}$. The double integral is to be taken over the surface of the meniscus $R$. For $|\Delta \psi| \ll 1$, we have

$$
\begin{array}{r}
W[\psi(x, y)]=\frac{1}{2} \iint_{R}\left(\rho g \psi^{2}+\gamma|\nabla \psi|^{2}\right) d x d y \\
+\int_{\Gamma} \phi(s) d s .
\end{array}
$$

Suppose $\psi^{*}(x, y)$ and $\phi^{*}(s)$ are chosen such that

$$
\psi^{*}(x, y)=\psi(x, y)+\epsilon \chi(x, y)
$$

and $\phi^{*}(s)=\phi(s)+\epsilon \gamma \phi_{0}(s) \chi(s)$, where $\epsilon$ is a very small number. Then the variation in the total energy, $\delta W=W\left[\psi^{*}(x, y)\right]-W[\psi(x, y)]$, is given by

$$
\begin{array}{r}
\delta W=\epsilon\left[\iint_{R}(\rho g \psi \chi+\gamma \nabla \psi \cdot \nabla \chi) d x d y\right. \\
\left.+\gamma \int_{\Gamma} \phi_{0} \chi d s\right]+O\left(\epsilon^{2}\right),
\end{array}
$$

where $O\left(\epsilon^{2}\right)$ represents the term of order $\epsilon^{2}$. The term $\gamma \nabla \psi \cdot \nabla \chi$ may be replaced by use of the identity $\nabla \cdot(\chi \nabla \psi)=\nabla \chi \cdot \nabla \psi+\chi \nabla^{2} \psi . \quad$ Furthermore,

$$
\nabla \cdot(\chi \nabla \psi)=\frac{\partial Q}{\partial x}+\frac{\partial P}{\partial y},
$$

where $P=\chi \psi_{y}, Q=\chi \psi_{x}$ and $\psi_{x}, \psi_{y}$ are partial derivatives of $\psi$ with respect to $x$ and $y$. Then by Stokes' theorem

$$
\iint_{R}\left(\frac{\partial Q}{\partial x}+\frac{\partial P}{\partial y}\right) d x d y=\int_{\Gamma}(-P d x+Q d y)
$$

eq (5) becomes

$$
\begin{aligned}
\delta W=\epsilon \iint_{R}\left(\rho g \psi-\gamma \nabla^{2} \psi\right) \chi d x d y & +\epsilon \int_{\Gamma} \gamma \phi_{0} \chi d s \\
& +\epsilon \int_{\Gamma} \gamma \chi\left(\psi_{x} d y-\psi_{y} d x\right) .
\end{aligned}
$$

In terms of the normal and tangential directions at $\Gamma$, the differential elements $d x, d y$ and the partial derivatives $\psi_{x}, \psi_{y}$ become $-\sin \theta d s, \cos \theta d s$,

$$
\frac{\partial \psi}{\partial n} \cos \theta \text { and } \frac{\partial \psi}{\partial n} \sin \theta,
$$

respectively, so that $\psi_{x} d y-\psi_{y} d x$ can be written as $\frac{\partial \psi}{\partial n} d s$. Thus,

$$
\begin{aligned}
\delta W=\epsilon \iint_{R}\left(\rho g \psi-\gamma \nabla^{2} \psi\right) \chi & d x d y \\
& +\epsilon \gamma \int_{\Gamma}\left(\phi_{0}+\frac{\partial \psi}{\partial n}\right) \chi d s
\end{aligned}
$$

The necessary condition that $W$ is a minimum is $\delta W=0$ for arbitrary variations $\chi(x, y)$ [7]. Hence, we obtain the equations

and

$$
\nabla^{2} \psi(x, y)-\frac{1}{a^{2}} \psi(x, y)=0
$$

$$
\frac{\partial \psi}{\partial n}+\phi_{0}=0
$$

where $a^{2}=\gamma / \rho g$. Solutions of eq (7) give functions that minimize $W$, while eq (8) provides the condition for the evaluation of the constants of integration. It is eq (7) which is now pertinent.

Since the gas-liquid interface is taken to be a surface of revolution, $\psi$ is a function of $z$ alone and eq (7) transformed to cylindrical coordinates $(z, \theta)$ becomes

$$
\frac{d^{2} \psi(z / a)}{d z^{2}}+\frac{1}{z} \frac{d \psi(z / a)}{d z}-\psi(z / a)=0
$$

A solution of eq (9) is

$$
\psi(z / a)=C_{1} I_{0}(z / a)
$$

where $I_{0}(z / a)$ is the modified Bessel function of the 
first kind and zero order. The meniscus height $h$ is given by the difference of $\psi$ at $z=0$ and $z=r$, and eq (10) becomes

$$
\psi(z / a)= \pm \frac{h I_{0}(z / a)}{1-I_{0}(r / a)}
$$

where the plus and minus signs refer to capillary depression and rise, respectively. The capillary depression $C=\psi(0)$ (see figure 1) is linearly proportional to the meniscus height, $h$ for a specific value of the ratio $r / a$. Differentiation of eq (10) gives

$$
\nabla \psi(z / a)= \pm \frac{h I_{1}(z / a)}{a\left[1-I_{0}(r / a)\right]}
$$

where $I_{1}(z)$ is the modified Bessel function of the first kind and order one. The condition for validity of linear behavior between $C$ and $h$ is that $|\nabla \psi(r / a)| \ll 1$.

\section{Results and Discussion}

In eq (10), vertical displacements of the meniscus are taken with respect to a carefully selected planar surface such that the value of the capillary depression is given by $C$. The condition that a meniscus be nearly planar implies that $h \ll r$. The condition that only menisci of capillary dimensions are to be considered requires that $0<r / a \leqq 1$, since $a \sim 2 \mathrm{~mm}$ for mercury. However, larger values of $r / a$ are admissible so long as the condition that the meniscus be nearly planar is met.

Table 1 gives capillary depressions $C=\psi(0)$, from eq (11) for some values of $r / a$. Table 2 gives a comparison of the capillary depressions predicted by eq (11) to those obtained from numerical solutions of Laplace's equation as performed by Akiyama, Hashimoto, and Nakayama [5], and Gould and Vickers [6]. Table 2 shows that results of this paper are in fair agreement with those obtained by numerical methods.

Figure 2 shows the dependence of capillary depressions on meniscus heights for some values of $r / a$. The solid lines are the linear behavior pre-

TABLE 1. Ratio of the capillary depression to the meniscus height of a nearly planar mercury meniscus for some values of $\mathrm{r} / \mathrm{a}$

\begin{tabular}{c|c|c|c|c|c|c|c|c}
\hline \hline$r / a \ldots \ldots \ldots \ldots . .$. & .20 & .30 & .40 & .50 & .60 & .70 & .80 & .90 \\
\hline Ratio........... & 100 & 44.2 & 24.7 & 15.7 & 10.9 & 7.91 & 6.01 & 4.69 \\
\hline
\end{tabular}

TABLE 2. Comparison of capillary depressions for a meniscus height

\begin{tabular}{|c|c|c|c|c|c|}
\hline$r / a$ & $A H N^{*}$ & $\mathrm{GV}^{* *}$ & This work & Difference & $|\nabla \psi|$ \\
\hline & $\mathrm{mm} \mathrm{Hg}$ & $m m ~ H g$ & $m m \mathrm{Hg}$ & Percent & \\
\hline .34 & 3.34 & & 3.43 & 2.9 & .3 \\
\hline .543 & & 1.32 & 1.33 & 0.8 & .2 \\
\hline .815 & & .573 & .578 & 0.9 & .1 \\
\hline .96 & .417 & & .409 & 2.0 & .1 \\
\hline
\end{tabular}
of mercury equal to $0.1 \mathrm{~mm}$

*Values taken from Akiyama, et al. [5].

**Values taken from Gould and Vickers [6]. dicted by this paper. The dotted lines are loci of constant $\nabla \psi$. These dotted lines approach the axes asymptotically as $r / a$ approaches zero or becomes very large. The points represent values obtained from numerical solutions of Laplace's equation. This figure shows that within the validity of the assumptions, capillary depressions are linearly related to meniscus heights. The figure shows that this linear relationship compares fairly well with results obtained from numerical solutions of Laplace's equation for $|\nabla \psi|<0.5$ and $r / a \gtrsim 0.35$.

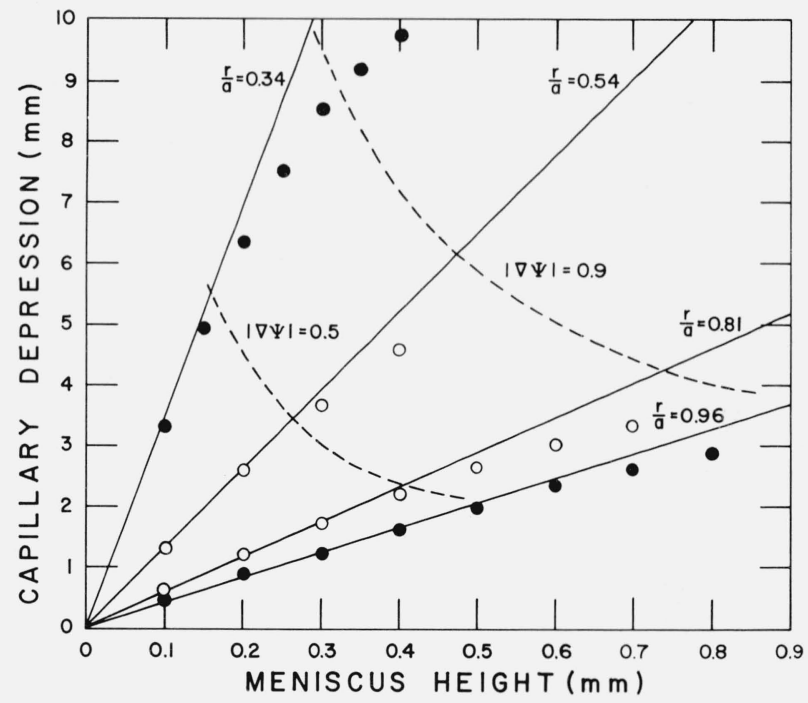

FIGURE 2. Capillary depression versus meniscus height for nearly planar mercury menisci.

Open and solid circles indicate values from numerical solutions of Laplace's equation: open circles, Gould and Vickers; solid circles, Akiyama, et al. Dotted lines indicate values
of $|\nabla \psi|$.

In summary, it has been shown that capillary depressions for nearly planar menisci are linearly related to their meniscus heights. Conditions for the validity of this behavior have been given.

The author is indebted to Stanley Ruthberg for suggesting this problem. The author is grateful to H.P. Waters for some helpful discussions in connection with this work.

\section{References}

[1] Moser, H., and Poltz, H., Instrumentkunde 65, 43 (1957).

[2] Laplace, P. S., Supplement à la Théorie de l'Action Capillaire, Transl. by Bowditch, Méchanique Celèste; Supplement to the Tenth Book (U.S. Naval Observatory, 1839), pp. 685-793.

[3] Blaisdell, E., J. Math. Phys. 19, 186 (1940).

[4] Bashford, F., and Adams, J. C., An Attempt to Test the Theories of Capillary Action (Cambridge University Press, Cambridge, England, 1883), pp. 1-73.

[5] Akiyama, Y., Hashimoto, H., and Nakayama, K., J. Vac. Soc. (Japan) 7, 12 (1964).

[6] Gould, F. A., and Vickers, T., J. Sci. Instr. 29, 35 (1952).

[7] Gelfand, I. M., and Formin, S. V., Calculus of Variations, Ch. 1 (Prentice-Hall, Inc., New Jersey, USA, 1963).

[8] Goodrich, F. C., Proc. Roy. Soc. A260, 481 (1961).

(Paper 73A6-574) 